\title{
Particle Confinement in Realistic 3D Rotamak Equilibria
}

\author{
P. M. Bellan \\ California Institute of Technology, Pasadena, California 91125 \\ (Received 6 February 1989)
}

\begin{abstract}
The rotating magnetic field $b_{\omega}$ of a rotamak tilts the effective flux surface seen by electrons (but not ions). Electrons are imperfectly confined to these tilted flux surfaces, but are energetically confined in a 3D potential well bounded by an untilted flux surface. When $e b_{\omega} / m_{e} v_{e i} \approx r_{0} / \delta_{\text {skin }}$ the tilt is minimized and the well depth corresponds to MHD pressure balance.
\end{abstract}

PACS numbers: $52.55 . \mathrm{Hc}, 52.20 . \mathrm{Dq}$, 52.25. Fi, 52.50.Dg

Steady-state toroidal current $I_{\theta}$ and axisymmetry are expected to confine plasma in a steady-state toroidal magnetic fusion reactor as follows: $I_{\theta}$ produces poloidal magnetic fields with associated toroidally nested flux surfaces $\Psi(r, z)$ while axisymmetry constrains particles to stay on a unique flux surface. The rotamak, ${ }^{1-7}$ an unorthodox toroidal plasma confinement device, employs a magnetic field $b_{\omega}$ rotating at a low frequency $\omega$ to give a net toroidal drift velocity $u_{\theta}$ to electrons (but not ions) and so provide ${ }^{8}$ the steady-state current

$$
I_{\theta}=5 e P / 8 m_{e} v_{e i} \omega \pi r_{0}^{2},
$$

where $P$ is the rotating-field input power, $v_{e i}$ is the electron-ion collision frequency, and $r_{0}$ is the major radius. Equation (1) was derived ${ }^{8}$ assuming that the solution to the Grad-Shafranov pressure balance equation is the analytically tractable, yet realistic, Solov'ev equilibri$\mathrm{um}^{9}$

$$
\Psi(r, z)=\Psi_{0} r^{2}\left(2 r_{0}^{2}-r^{2}-\alpha^{2} z^{2}\right) / r_{0}^{4},
$$

where $\Psi_{0}$ is the flux on the magnetic axis $\left(r=r_{0}, z=0\right)$, the outer intersection of the separatrix with the midplane $z=0$ occurs at $R_{\text {sep }}=\sqrt{2} r_{0}$, and $\alpha$ is the separatrix's ellipticity.

Equation (1) indicates very efficient current drive (i.e., large $\left.I_{\theta} / P\right)$ at large electron temperature $T_{e}$ where $v_{e i}$ becomes very small. Furthermore, Ref. 8 demonstrated that Eq. (1) predicts reasonably accurately the $I_{\theta}$ measured in two rotamak experiments ${ }^{4,6}$ sufficiently well diagnosed to make a comparison. However, the actual $I_{\theta}$ 's in Refs. 4 and 6 were low because $T_{e}$ was low $(\approx 6-10$ eV).

$T_{e}$ was low in Refs. 4 and 6 despite the injection of substantial power (10-50 kW) into small volumes; thus, energy confinement is clearly a critical issue for rotamaks. In fact, Ref. 8 showed that the confinement of the prolate rotamak of Ref. 6 was only slightly better than what would be attained if there were no magnetic confinement at all and instead the plasma simply drifted out at the ion acoustic velocity, whereas the confinement of the nearly spherical rotamak of Ref. 4 was only about an order of magnitude better than acoustic confinement. This suggests that prolate $(\alpha \ll 1)$ geometry provides intrinsically worse confinement than spherical $(\alpha \approx 1)$ geometry. The magnetic fields in these experiments were very low, making the ion Larmor orbits so large that ions were unconfined. In contrast, electron Larmor orbits were small and the electrons ought to have been confined to magnetic flux surfaces $\Psi(r, z)=$ const. This observation of near-acoustic confinement (i.e., electrons confined only by the retarding ambipolar potential resulting from the charge separation setup when they try to escape) suggests that the electrons in Refs. 4 and 6 were not well confined to flux surfaces [however, the short mean free path in Refs. 4 and 6 (order of the device size) somewhat complicates this interpretation].

Concern has been expressed that the rotating magnetic field opens field lines, and so destroys particle confinement. In response to this concern, Hugrass and Turley ${ }^{10}$ analyzed particle orbits in a field consisting of the sum of the equilibrium field associated with a Solov'ev flux function, $b_{\omega}$, and its associated electric field, and an ambipolar electrostatic field. They showed for this particular case that (i) when transformed to the rotating frame, the equations of motion describe particle motion in a combination of a steady magnetic field and an electrostatic potential implying that the energy in the rotating frame is constant, and (ii) electrons are in a potential well in the rotating frame, whereas ions are in a potential hill; (iii) they provided plots of numerically integrated particle orbits demonstrating confinement.

This Letter, using a Hamiltonian-Lagrangian analysis, presents important new confinement effects. We begin by assuming ${ }^{10}$ (i) that the rotating field is fully penetrated, ${ }^{11}$ (ii) that it does indeed cause a net electron toroidal drift constituting an $I_{\theta}$, and (iii) that an externally produced vertical field is added to balance the $I_{\theta}$ force so that the net equilibrium field has surfaces of poloidal flux $\Psi(r, z)$ given by Eq. (2). The total dc magnetic field is related to $\Psi$ by

$$
\mathbf{B}=(2 \pi)^{-1} \nabla \Psi \times \nabla \theta .
$$

Also, $\Psi$ is related to the toroidal vector potential as $\Psi(r, z)=2 \pi r A_{\theta}(r, z)$.

We calculate particle orbits in the sum of the equilibrium magnetic field given by Eq. (3) and the fully penetrated rotating rotamak electric and magnetic fields

$$
\begin{aligned}
& \tilde{E}_{z}=\omega r b_{\omega} \cos (\theta-\omega t) \\
& \tilde{\mathbf{B}}=b_{\omega}[\hat{\mathbf{r}} \cos (\theta-\omega t)-\hat{\boldsymbol{\theta}} \sin (\theta-\omega t)]
\end{aligned}
$$


Here $\tilde{\omega}_{c i} \ll \omega \ll \tilde{\omega}_{c e}$ and $\tilde{\omega}_{c i}=e b_{\omega} / m_{i}, \tilde{\omega}_{c e}=e b_{\omega} / m_{e}$ are cyclotron frequencies defined with respect to the rotating field strength. Also, the fields of Eq. (4) can both be derived from the vector potential

$$
\tilde{A}_{z}=r b_{\omega} \sin (\theta-\omega t) \text {. }
$$

Equation (4) gives the vacuum fields produced by the rotating field coils; Jones and Hugrass ${ }^{11}$ showed that these are also the fields when $u_{\theta}$ is synchronous with the rotating field, because then $\tilde{E}_{z}$ vanishes in the electron frame and so the electron fluid cannot do any shielding. This fully penetrated regime occurs when the accelerating toroidal force associated with the rotating field is much stronger than the frictional drag force, and corresponds ${ }^{11}$ to the condition

$$
\tilde{\omega}_{c e} / v_{e i} \geq a / \delta_{\text {skin }},
$$

where $a \sim r_{0}$ is the minor radius and $\delta_{\text {skin }}=\left(2 m_{e} v_{e i}\right)$ $\left.\mu_{0} \omega n e^{2}\right)^{1 / 2}$ is the classical skin depth.

Defining the rotating-frame toroidal angle $\xi \equiv \theta-\omega t$, we see that $\dot{\theta}=\dot{\xi}+\omega$. In this frame the Lagrangian of a particle with charge $q$ is

$$
\begin{aligned}
L=\frac{1}{2} m\left[\dot{r}^{2}+r^{2}(\dot{\xi}+\omega)^{2}+\dot{z}^{2}\right] & \\
& +q r(\dot{\xi}+\omega) A_{\theta}+q \dot{z} r b_{\omega} \sin \xi .
\end{aligned}
$$

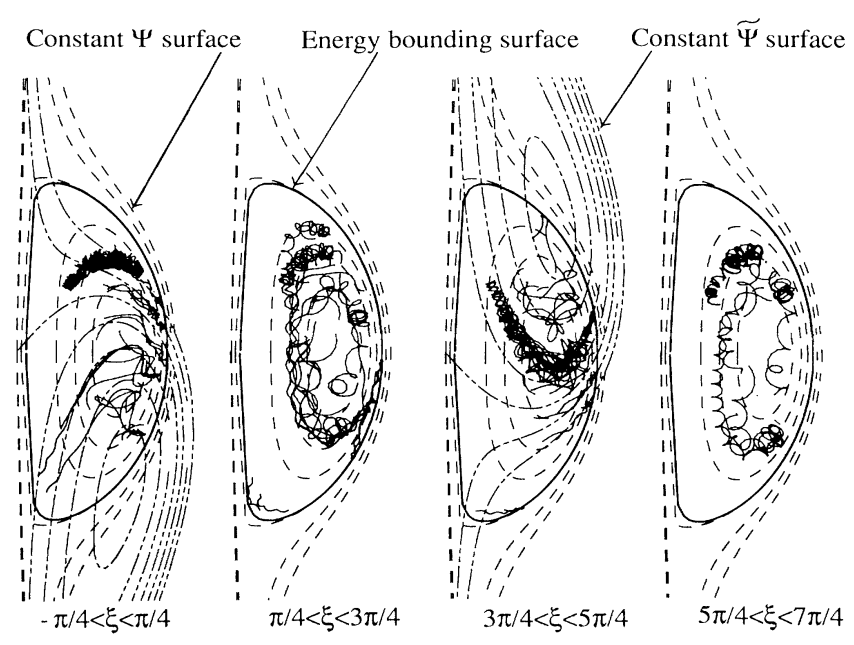

FIG. 1. Numerically integrated electron orbit in minor cross section of rotating frame; dashed lines are untilted flux surfaces [Eq. (3)], solid line is energy bounding surface [Eq. (9)], dash-dotted lines are tilted flux surfaces [Eq. (13)]; parameters (chosen to be similar to Ref. 4) are $T_{e}=10 \mathrm{eV}, \bar{B}=40 \mathrm{G}$, $b_{\omega}=17 \mathrm{G}, r_{0}=8 \mathrm{~cm}, \alpha=0.8, f=1 \mathrm{MHz}$, and duration of integration corresponds to $6 \mu \mathrm{sec}$.

It is more convenient to use the Hamiltonian $H=\sum P_{j} \dot{Q}_{j}-L$ where $P_{r} \equiv \partial L / \partial \dot{r}=m \dot{r}, \quad P_{\xi} \equiv \partial L / \partial \dot{\xi}=m r^{2}(\dot{\xi}+\omega)$ $+q r A_{\theta}(r, z)$, and $P_{z} \equiv \partial L / \partial \dot{z}=m \dot{z}+q r b_{\omega} \sin \xi$. Evaluation of the Hamiltonian gives

$$
H(r, \xi, z, \dot{r}, \dot{\xi}, \dot{z})=\frac{1}{2} m\left(\dot{r}^{2}+r^{2} \dot{\xi}^{2}+\dot{z}^{2}\right)-\frac{1}{2} m r^{2} \omega^{2}-q r \omega A_{\theta}
$$

or, in terms of canonical variables,

$$
H\left(r, \xi, z, P_{r}, P_{\xi}, P_{z}\right)=\frac{P_{r}^{2}}{2 m}+\left[\frac{\left(P_{\xi}-q r A_{\theta}\right)^{2}}{2 m r^{2}}-\omega P_{\xi}\right]+\frac{\left(P_{z}-q r b_{\omega} \sin \xi\right)^{2}}{2 m} .
$$

Since $L$ does not explicitly depend on time, $H=$ const; i.e., the energy in the rotating frame is a constant ${ }^{10}$ of the motion. Also, from Eq. (8a) we see that $H=T+V$, where $T=\frac{1}{2} m\left(\dot{r}^{2}+r^{2} \dot{\xi}^{2}+\dot{z}^{2}\right)$ is the kinetic energy in the rotating frame, and $V(r, z)=-\frac{1}{2} m r^{2} \omega^{2}$ $-q r \omega A_{\theta}(r, z)$ is the potential energy in the rotating frame. If $V(r, z)$ has a minimum, then a particle can be trapped in the well centered about this minimum. Since the electron mass is so small, the electron centrifugal term is negligible. Using $q_{e}=-e$, the electron potential is just $V \approx e f \Psi(r, z)$ where $f=\omega / 2 \pi$; this indeed has a well shape, since $\Psi(r, z)$ has a minimum on the magnetic axis $r=r_{0}, z=0$. The maximum electron excursion up the well "side" occurs when all the electron kinetic energy has turned into potential energy, i.e., when

$$
T_{0}+V_{0} \approx e f \Psi(r, z)
$$

where $T_{0}$ and $V_{0}$ are the respective initial electron kinetic and potential energies. Figure 1 shows electron orbits obtained by numerical integration of the Lorentz-force equation using the fields of Eqs. (2)-(4); parameters were chosen to simulate Ref. 4 . The minor cross section is plotted in four segments, each corresponding to a toroidal quadrant in the rotating frame. The electron trajectory lies inside the volume bounded by the flux surface defined by Eq. (9). This energy bonding surface (solid line in the Fig. 1 quadrant plots) depends on both the electron's initial position (which determine $V_{0}$ ) and its initial velocity (which determines $T_{0}$ ). The orbit in Fig. 1 clearly stays within this bounding flux surface; also, when initial conditions were varied to give a different bounding flux surface, the electron orbit stayed within this new bounding surface. For ions, the potential energy reverses sign, so that the ions are on a potential hill. $^{10}$

A particle can be confined by (i) being in a potential well (energy-based confinement), or, alternatively, by (ii) conservation of canonical momentum (momentumbased confinement). That these two mechanism differ completely is demonstrated by the everyday example of momentum-based confinement where a spinning top stays upright due to angular momentum conservation and yet is clearly not energetically confined, since its 
equilibrium position is at a potential maximum.

Particle confinement to flux surfaces is a form of momentum-based confinement and so relies on symmetry; to observe the effect of the rotating field on this confinement consider now the $\xi$ component of Hamilton's equations, $d P_{\xi} / d t=-\partial H / \partial \xi$, or

$$
d\left[m r^{2}(\dot{\xi}+\omega)+q r A_{\theta}\right] / d t=q r \dot{z} b_{\omega} \cos \xi .
$$

Recalling that the particle's gyroradius $\sim \sqrt{m}$, we see that the particle's guiding-center trajectory is easily found by letting $m \rightarrow 0$ (massless particle) in Eq. (10), giving

$$
d \Psi / d t=2 \pi r \dot{z} b_{\omega} \cos \xi .
$$

Thus, if $b_{\omega}=0$ we obtain the familiar result that the particle's guiding center is confined to a flux surface $\Psi$ (in fact, demonstration of this provided a useful check for our numerical code). However, if $b_{\omega} \neq 0$, then toroidal symmetry is destroyed, and Eq. (11) indicates that $d \Psi / d t \neq 0$ so that the particle is not confined to an equilibrium flux surface $\Psi$.

If somewhere in the particle's orbit $z$ changes more rapidly than both $r$ and $\xi$, then over this portion of the orbit we can approximate $r$ and $\xi$ as being constant and integrate Eq. (11) to obtain

$$
\tilde{\Psi}=\Psi-2 \pi r z b_{\omega} \cos \xi \approx \text { const } .
$$

Using Eq. (2) in Eq. (12) we see that the surfaces of constant $\tilde{\Psi}$ are given by the curves

$$
z=\frac{2 \delta \cos \xi \pm\left\{(2 \delta \cos \xi)^{2}+2 \alpha^{2}\left[r^{2}\left(1-r^{2} / 2\right)-\tilde{\Psi} / 2\right]\right\}^{1 / 2}}{\alpha^{2} r},
$$

where, for clarity, all lengths have been normalized to $r_{0}$, the flux has been normalized to $\Psi_{0}, \delta=b_{\omega} / \bar{B}$, and $\bar{B}=-\Psi_{0} / \pi r_{0}^{2}$ is the equilibrium field measured on the separatrix at $z=0, r=2^{1 / 2} r_{0}$. The constant $-\tilde{\Psi}$ surfaces given by Eq. (13) are drawn for $\xi=0$ and $\pi$ as the dashdotted lines in the first and third quandrants of Fig. 1. These constant $-\tilde{\Psi}$ surfaces are tilted flux surfaces in the rotating frame, since they are downwardly shifted for $\xi=0$, and upwardly shifted for $\xi=\pi$. The tilt is caused by the rotating field enhancing (diminishing) the strength of the horizontally directed equilibrium field on the top (bottom) at $\xi=0$ and vice versa at $\xi=\pi$.

Electrons, moving with thermal velocities much faster than the rotating field velocity, tend to follow the essentially poloidal magnetic field, and so see $\cos \xi$ as an approximately steady-state term. Hence, electron guiding centers are confined (within a gyroradius) to the surface $\tilde{\Psi} \approx$ const for the orbit portion where $\dot{z}$ is large (i.e., this excludes turning points in $z$ where $\dot{z}=0$ ). Since $\tilde{\Psi}$ is an imperfect constant of the motion, electrons are imperfectly attached to constant $-\tilde{\Psi}$ surfaces, and slowly shift from one surface to another, filling up the entire volume bounded by the surface determined by Eq. (9). Examination of Fig. 1 shows that the electrons do indeed follow the $\tilde{\Psi}$ surfaces and also stay on a given surface for only a limited time.

If the ion thermal velocity is much lower than the rotating field velocity, the term $\cos \xi$ appears highly oscillatory to ions. This term consequently averages to zero so far as ions are concerned, and so the ion gyroradii are confined to the original untilted flux surface $\Psi$.

When there is no rotating field (no tilt), the separatrix corresponds to the $\Psi(r, z)=0$ surface. We quantify the tilt by examining the shift in $z$ of the separatrix. The curve for the shifted separatrix is found by setting $\tilde{\Psi}=0$ in Eq. (13); if we assume that $\delta \ll 1$, we find that the curve for the shifted separatrix is

$$
z \approx \frac{2 \delta \cos \xi}{\alpha^{2} r} \pm \frac{\left(2-r^{2}\right)^{1 / 2}}{\alpha}
$$

From Eq. (14) we see that for small $\delta$ the shift in $z$ of the separatrix due to the tilt is given by $\Delta z \approx 2 \delta / \alpha^{2}$, since the maximum $z$ of the separatrix occurs when $r \approx 1$. The $z$ height of the untilted separatrix is $z_{\max }$ $\approx 1 / \alpha$ so that the normalized shift is $\Delta z / z_{\max } \approx 2 \delta / \alpha$. This normalized shift is large for prolate (i.e., $\alpha \ll 1$ ) geometries; this is probably why the $\alpha=0.2$ plasma of Ref. 6 had $^{8}$ much worse confinement than the $\alpha=0.8$ plasma of Ref. 4.

When the plasma is highly tilted, there is a nearly free flow of electrons and thus heat from the central region of the plasma to the outer surface, since (i) the tilted flux surfaces intersect the wall and, additionally, (ii) the electrons are imperfectly confined to flux surfaces. Collisions make the electron velocity distribution Maxwellian, creating some energetic electrons. If these electrons have a total energy $T_{0}+V_{0}>0$ (which will occur when $T_{0}$ exceeds the well depth ef $\Psi_{0}$ ) then their bounding surface will correspond to an untilted flux surface outside the untilted separatrix. For example, Ref. 6 had $\Psi_{0}=3.6 \times 10^{-6} \mathrm{~Wb}$ and $f=1 \mathrm{MHz}$, so that all electrons with $T_{0}$ greater than $f \Psi_{0}=3.6 \mathrm{eV}$ would have bounding surfaces outside the untilted separatrix, and since $T_{e}$ was $6 \mathrm{eV}$ most electrons would be in this class. Such flux surfaces outside the separatrix are unbounded in the $z$ direction, and so an electron that attains kinetic energy greater than ef $\Psi_{0}$ could be lost by flowing out in the $z$ direction inside a cylindrical surface given by Eq. (9). However, if these electrons have a finite magnetic moment, they will reflect off a magnetic mirror at some value of $z$ (since $B_{z}$ increases with $|z|$ outside the separatrix) resulting in a mirror-confined plasma elongated in the $z$ direction beyond the separatrix. This probably explains the recent puzzling observation ${ }^{7}$ in the UCLA Racetrack rotamak of significant plasma density extending in the $z$ direction $50 \%$ beyond the separatrix.

Summarizing, for electrons (i) the rotating field causes a rotating tilt $\sim b_{\omega} / \alpha \bar{B}$ of the effective flux sur- 
faces, and (ii) electrons are imperfectly confined to these tilted flux surfaces, and (iii) the electrons are confined with a $3 \mathrm{D}$ potential well $\approx e f \Psi(r, z)$, which has the shape of an untilted flux surface. Electrons rattle around in this well, shifting from one tilted flux surface to another until their orbit has filled up with entire potential well volume. For ions, (i) providing the ion velocity is sufficiently slow compared to the rotation of the tilted flux surface, the ions see only the average of the tiIted flux surface, which is just the original untilted flux surface and so are confined in the usual way, and (ii) the potential well reverses sign and so becomes a potential hill. Hence, ions are confined by momentum conservation, not energy conservation, whereas the opposite is true for electrons. Geometrically, the electrons are confined to a volume (the potential well volume), whereas ions are confined to a surface (the flux surface).

Small $b_{\omega} / \alpha \bar{B}$ would minimize the tilt. This could be achieved if the plasma were very hot so that a substantial $I_{\theta}$ and hence large $\bar{B}$ could be produced with a small $b_{\omega}$. However, in all experiments to date the plasma was cold so that $b_{\omega} / \alpha \bar{B} \approx 0.1-0.5$, making the tilt very important. Durance et al. ${ }^{4}$ observed for the range of parameters obtainable in their experiment (cf. their Fig. 6) that the driven toroidal current $I_{\theta}$ and hence $\bar{B}$ scaled as $P^{1 / 2}$. Since $b_{\omega}$ also scales as $P^{1 / 2}$, this means that the tilt magnitude $b_{\omega} / \alpha \bar{B}$ stayed constant when $P$ was increased, making it impossible to achieve better confinement simply by increasing $P$.

Let us now examine to what extent $\delta$ can be minimized and the related issue of how deep an energy well can be created. Minimizing $\delta$ requires maximizing the rotating-field coils' rf loading resistance $R_{\text {load }}$, because $I_{\theta} \sim P \sim I_{c}^{2} R_{\text {load }}$ and $I_{c} \sim b_{\omega}$, where $I_{c}$ is the ac current in the rotating-field coils. This required maximization of $R_{\text {load }}$ is obtained when ${ }^{8}$ the equal sign is used in Eq. (6) and the corresponding $I_{\theta}$ is ${ }^{8}$ approximately half what would be obtained if all electrons moved synchronously with the rotating field; i.e., $I_{\theta}$ is given by

$$
I_{\theta} \approx 2 \sqrt{2} n r_{0}^{3} e \omega / 3 \alpha
$$

Integration of the Solov'ev current density $J_{\theta}=4 \Psi_{0}(1$ $\left.+\alpha^{2} / 4\right) r / \pi \mu_{0} r_{0}^{4}$ over the mirror cross section up to the separatrix gives $I_{\theta}=16 \sqrt{2} \Psi_{0}\left(1+\alpha^{2} / 4\right) / 3 \pi \mu_{0} r_{0} \alpha$. The flux corresponding to Eq. (15) is thus $\Psi_{0}=\pi n r_{0}^{4} e \omega \mu_{0} / 8$ $\times\left(1+\alpha^{2} / 4\right)$ making the depth of the energy well (for minimum tilt)

$$
e f \Psi_{0}=n e^{2} r_{0}^{4} \omega^{2} \mu_{0} / 16\left(1+\alpha^{2} / 4\right),
$$

and we see that confinement of high-temperature plasmas may be obtained most easily by making $r_{0}$ larger. If we eliminate $\omega$ in Eq. (16) using Eq. (6), we find that the minimum obtainable tilt is

$b_{\omega} / \bar{B} \approx 4\left(1+\alpha^{2} / 4\right) v_{e i} / \tilde{\omega}_{c e} \approx 4\left(1+\alpha^{2} / 4\right) \delta_{\text {skin }} / r_{0}$,

suggesting the desirability for using auxiliary heating to increase $T_{e}$ and so reduce the tilt. We note that the Grad-Shafranov equation also gives a limit on pressure confinement (obtained by equating the $J_{\theta}$ expressed in terms of flux to the $J_{\theta}$ expressed in terms of pressure); this MHD limit turns out to be identical to Eq. (16) [except for a factor of 2 , probably due to the approximations in the derivation of Eq. (16)]. It is consequently not possible to make the Eq. (16) well depth significantly deeper than $k T_{e}$, since if one tried, there would result excess MHD confining force which would pinch the plasma to a smaller radius.

Finally, we postulate that the helical Rhythmac ${ }^{12}$ configuration probably has a related, but helical-like tilt; however, due to the Rhythmac's helical symmetry, it is likely that electrons will be confined in stellarator fashion to rotating helical flux surfaces, and unlike the rotamak will not rattle around in a volume.

This work was supported by U.S. DOE Grant No. DE-FG03-86ER53232.

'W. N. Hugrass et al., Phys. Rev. Lett. 44, 1679 (1980).

${ }^{2}$ G. Durance et al., Phys. Rev. Lett. 48, 1252 (1982).

${ }^{3}$ I. R. Jones, Comments Plasma Phys. Controlled Fusion 10, 115 (1986).

${ }^{4}$ G. Durance et al., Plasma Phys. Controlled Fusion 29, 227 (1987).

${ }^{5}$ G. A. Collins et al., Nucl. Fusion 28, 255 (1988).

${ }^{6}$ A. J. Knight, Ph.D. thesis, Flinders University, 1988 (unpublished).

${ }^{7}$ H. R. Zwi et al., Bull. Am. Phys. Soc. 33, 2002 (1988).

${ }^{8}$ P. M. Bellan (to be published).

${ }^{9}$ L. S. Solov'ev, Rev. Plasma Phys. 6, 239 (1976).

${ }^{10} \mathrm{~W}$. N. Hugrass and M. Turley, J. Plasma Phys. 37, 1 (1987).

${ }^{11}$ I. R. Jones and W. N. Hugrass, J. Plasma Phys. 26, 441 (1981).

${ }^{12}$ M. Dutch and A. L. McCarthy, Plasma Phys. Controlled Fusion 28, 695 (1986). 